\title{
Dynamic Performance Analysis of a Switched Reluctance Generator 6x4 for Wind Energy Application
}

\author{
G.P.Viajante, E.N.Chaves, M.A.A.Freitas, C.A. Queiroz, J.A.Santos, S.B. Silva, O.C.Souto, \\ M.E,Oliveira and L. G. W. da Silva
}

Federal Institute of Education, Science and Technology of Goiás

Campus of Itumbiara - Goiás, (Brazil)

Phone/Fax number: 5564 343152005, e-mail: ghunterp@gmail.com

\begin{abstract}
This paper presents a Switched Reluctance Generator dynamic performance analysis as a function of excitation voltage and magnetization angles. A machine mathematical model is presented including the magnetic circuit saturation through the inductance representation of the phases by Fourier series expansion. The equations for the linkage flux, coenergy and electromagnetic torque are obtained from the inductance expression. The simulation was performed in Matlab/Simulink. Simulated and experimental results are presented considering the generator driven under three aspects: variable excitation voltage and fixed rotor speed, fixed excitation voltage and variable rotor velocity and finally varying the generator magnetization angles.
\end{abstract}

\section{Key words}

Switched Reluctance Generator, Half Bridge Converter and Dynamic Performance.

\section{Introduction}

Renewable energy sources, such as the strength of waters, wind or solar energy and fossil resources are among the fuels used to generate electricity however, rotating electric generators are responsible for almost all of the electrical energy produced so the importance of electric machines in the global context of energy management and sustainability is unparalleled.

Therefore, it is evident that the understanding of the characteristics and operational demands of electric machines must be under continuous investigation, in constant search for better flexibility rates, efficiency and operational performance.

In this context the Switched Reluctance Machine (SRM) has been the object of study by researchers worldwide mainly due to the advent of power electronics and the rapid development of high performance and low cost microprocessor systems, which have contributed in a systematic way so that the SRM became a strong candidate to be used as a generator due to its intrinsic characteristics such as robustness, no windings and permanent magnets in the rotor, good weight to power ratio, high starting torque, ability to operate in the absence of one of the phases in polyphase machines, low manufacturing cost and ease of design. [1][2][3].

The wind power market is dominated by synchronous and asynchronous machines, but the Switched Reluctance Generator (SRG) has been the target of several scientific-technological investigations, which point out the SRG as an alternative for such an application [4][5].

The wind has unstable conditions and the SRG can operate well under a wide range of varying speeds. This is the reason why research on wind SRG is intense [8].

\section{SRG Non-Linear Model}

Mathematical modeling for SRG dynamic analysis is described with reference to a machine single phase which can be represented as

$$
v=R i+\frac{\partial \lambda(i, \theta)}{d t}
$$

At a constant speed, the phase voltage equation is given by:

$$
v=R i+l \frac{d i}{d t}+e
$$

Where $v$ is the applied voltage, $i$ is the phase current, $R$ is the phase resistance, $e=\omega_{r} \partial \lambda(i, \theta) / \partial \theta$ is the back electromotive force, $l=\partial \lambda(i, \theta) / \partial i$ is the incremental phase inductance, $\theta$ is the rotor position.

The effects of magnetic saturation are considered in the machine model through the phase inductance analytical representation using Fourier series expansion [5]

The strategy for representing the inductance profile for different current values and rotor position is based on an approximation using a set of straight line segments as shown in Figure 1.

In order to facilitate the mathematical development, Figure 1 shows the inductance behavior of the winding in one of the phases for a given current value and 
considering that the rotor is displaced from a half-pitch of a pole.

Applying mathematical concepts for solving a periodic waveform the inductance expression of the prototype evaluated in this work can be determined as

$$
\begin{aligned}
& L(i, \theta)=\frac{1}{G} \sum_{j=1}^{m}\left(l_{(j+1)}\left(i, \alpha_{j}\right)+l_{j}\left(i, \alpha_{j}\right)\right)\left(\alpha_{(j+1)}-\alpha_{j}\right)+ \\
& \sum_{n=1}^{\infty}\left(\sum_{j=1}^{m}\left(l_{(j+1)}(i) A_{(j, n)}-l_{j}(i) B_{(j, n))}\right)\right) \cos \left(n P_{r} \theta\right)
\end{aligned}
$$

Where:

$$
\begin{aligned}
& A_{(j, n)}=\frac{2}{n \pi}\left(\sin \left(n P_{r} \alpha_{\left(j_{n}+1\right)}\right)+\frac{\cos \left(n P_{r} \alpha_{\left(j_{n}+1\right)}\right)-\cos \left(n P_{r} \alpha_{j}\right)}{n N_{r}\left(\alpha_{\left(j_{n}+1\right)}-\alpha_{j}\right)}\right) \\
& B_{(j, n)}=\frac{2}{n \pi}\left(\sin \left(n P_{r} \alpha_{j}\right)+\frac{\cos \left(n P_{r} \alpha_{(j+1)}\right)-\cos \left(n P_{r} \alpha_{j}\right)}{n N_{r}\left(\alpha_{\left(j_{n}+1\right)}-\alpha_{j}\right)}\right),
\end{aligned}
$$

$m$ is the straight line segment number, $n$ is the harmonic order, $P_{r}$ is the rotor pole number, $G=2 \pi / P_{r}$ é is the rotor pitch pole, $\theta$ is the rotor position, $l_{j}$ is the $j^{\text {th }}$ segment, $\alpha_{j}$ é is the inductance associated position [5].

A polynomial fit was used to represent the function $l_{j}\left(i, \alpha_{j}\right)$. The expression for $l_{j}\left(i, \alpha_{j}\right)$ is given as

$$
l_{j}\left(i, \alpha_{j}\right)=C_{3 j} i^{3}+C_{2 j} i^{2}+C_{1 j} i+C_{0 j}
$$

The expression for linkage flux comes from equations (3) through (6) and can be written as (7). The co-energy and torque can be written in the form of expressions (8) and (9).

The importance of analytically expressing the nonlinear relations of flux, current and position in the variable reluctance machine is that through the incremental inductance, the co-energy and torque can be analytically described and even bring forward the implementation of flux and torque observers.

$$
\begin{aligned}
& \lambda(i, \theta)=L(i, \theta) i= \\
& \frac{1}{G} \sum_{j=1}^{m}\left(\begin{array}{l}
\left(C_{3(j+1)}+C_{3 j}\right) i^{4}+\ldots+ \\
\left(C_{0(j+1)}+C_{0 j}\right) i
\end{array}\right)\left(\alpha_{(j+1)}-\alpha_{j}\right)+ \\
& \sum_{n=1}^{\infty}\left(\begin{array}{l}
\sum_{j=1}^{m}\left(C_{3(j+1)} i^{4}+\ldots+C_{0(j+1)} i\right) A_{(j, n)} \\
-\left(C_{3 j} i^{4}+\ldots+C_{0 j} i\right) B_{(j, n)}
\end{array}\right) \cos \left(n P_{r} \theta\right)
\end{aligned}
$$

$$
\begin{aligned}
& W^{\prime}(i, \theta)=\int_{0}^{i} \lambda(i, \theta) d i= \\
& \frac{1}{G} \sum_{j=1}^{m}\left(\begin{array}{l}
\left(\frac{C_{3(j+1)}+C_{3 j}}{5}\right) i^{5}+\ldots+ \\
\left(\frac{C_{0(j+1)}+C_{0 j}}{2}\right) i^{2}
\end{array}\right)\left(\alpha_{(j+1)}-\alpha_{j}\right)+ \\
& \sum_{n=1}^{\infty}\left(\sum_{j=1}^{m}\left(\begin{array}{l}
\left.\left.\left(\left(\frac{C_{3(j+1)}}{5}\right) i^{5}+\ldots+\left(\frac{C_{0(j+1)}}{2}\right) i^{2}\right) A_{(j, n)}\right)\right) \\
-\left(\left(\frac{C_{3 j}}{5}\right) i^{5}+\ldots+\left(\frac{C_{0 j}}{2}\right) i^{2}\right) B_{(j, n)}
\end{array}\right)\right) \cos \left(n P_{r} \theta\right)
\end{aligned}
$$

$$
\begin{aligned}
& T_{e_{\text {mag }}}=\frac{\partial W^{\prime}(i, \theta)}{\partial(\theta)}= \\
& =-n P_{r} \sum_{n=1}^{\infty}\left(\sum_{j=1}^{m}\left(\begin{array}{l}
\left.\left(\left(\frac{C_{3(j+1)}}{5}\right) i^{5}+\ldots+\left(\frac{C_{0(j+1)}}{2}\right) i^{2}\right) A_{(j, n)}\right) \\
-\left(\left(\frac{C_{3 j}}{5}\right) i^{5}+\ldots+\left(\frac{C_{0 j}}{2}\right) i^{2}\right) B_{(j, n)}
\end{array}\right)\right) \sin \left(n P_{r} \theta\right)
\end{aligned}
$$

At this point it is opportune noticing that expressions (3) to (9) include magnetic saturation, in other words they vary with current and position.

For any current value within the operating range at any given position, the instantaneous values of phase inductance, co-energy and torque can be obtained, making the mathematical model very suitable for dynamic calculations [5].

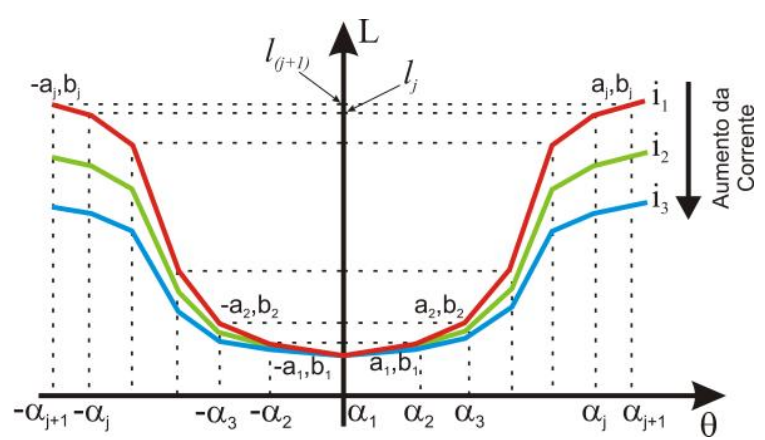

Fig. 1. Approximate representation of the inductance profile using "j" segments of straight lines.

Considering the viscous friction losses (D) and the moment of inertia $(\mathrm{J})$, the mechanical torque produced by the machine was calculated as

$$
T_{m}=T_{\text {emag }}-J \frac{d \omega}{d t}-D \omega
$$

From the presented equations the mathematical model which describes the SRG dynamics can be visualized in a state matrix form by 


$$
[v]=[X][i]+[Y][\dot{i}]
$$

Where $\mathrm{X}$ and $\mathrm{Y}$ are arrays which depend on machine constructing parameters.

Thus the system solution leads to a complete SRG analysis [5][8]

\section{Switching Strategy}

In this work the half bridge converter (HB) was used to drive the SRG. The working principle of this converter can be condensed into two stages, which are: excitation stage and free-wheeling stage in generation, whose topology and operation principle are explained in the following sections.

\section{Stage I - Excitation}

In stage I, as shown in Figure 2(a), the DC voltage bus that feeds the power circuit and the SRG is guaranteed by a single-phase or three-phase rectifier either one is well suited. Initially the magnetizing current is established closing S1 and S2 switches of the HB converter.

This current is responsible for creating the magnetizing flux in the core, which produces a restoring torque, resulting in a back electromotive force with an additive effect on the excitation voltage.

In this step, the two voltage producing sources contribute to the current growth. Typically, the energization of each phase is initiated before the total alignment between the stator and rotor poles.

This is done to ensure a reasonable current intensity during the period in which the inductance decreases and when the generator torque is produced. In this work, this angle is established in $-3^{\circ}$ (minus three degrees).

\section{Stage II - Freewheel in Generation}

This step happens when opening S1 and S2 switches. The voltage in the stator winding inverts its direction starting the process of transferring power to the load according to Figure $2 \mathrm{~b}$. In this region, due to the backelectromotive force, the current in the phase continues to increase until reaching its peak value.

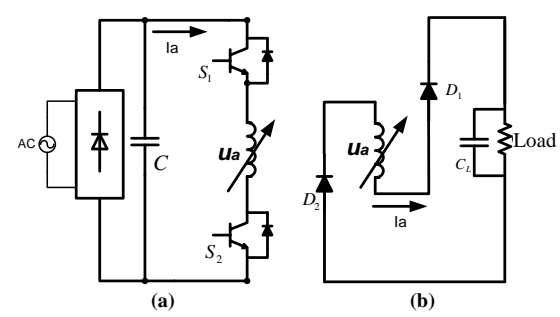

Fig. 2. Excitation (a); Free wheel of generation (b).

\section{Simulation Results}

The SRG was simulated under various operating regimes. Initially tests were carried out at constant speed.

In this case, the operating speed was set at $1500 \mathrm{rpm}$. The firing angle $\theta_{o n}$ is maintained fixed at $-3^{\circ}$ with respect to the alignment position and $\theta_{\text {off }}$ at $30^{\circ}$ with respect to $\theta_{o n}$. The excitation voltage was adjusted at $60 \mathrm{~V}$ and the load at $20 \Omega$. Figure 3 illustrates the curves for this situation.

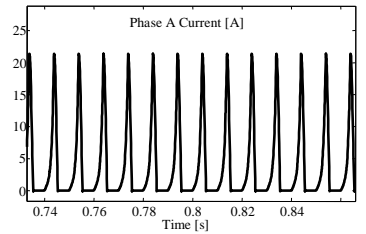

(a)

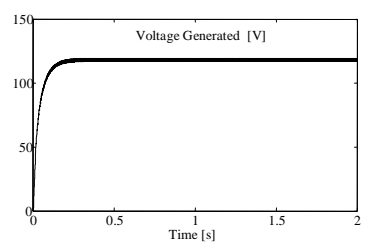

(c)

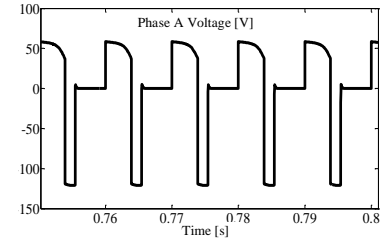

(b)

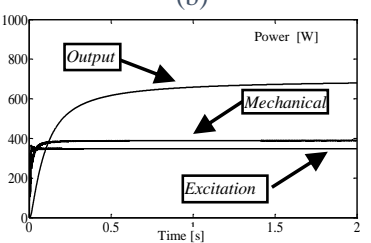

(d)
Fig. 3. Phase A Current (a) - Phase A Voltage

(b) - Voltage Generated (c) - Power (d)

Then the excitation voltage was changed to $80 \mathrm{~V}$. In this case Figure 4 shows the SRG behavior for this situation.

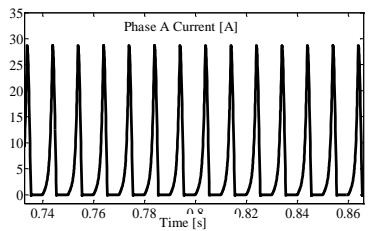

(a)

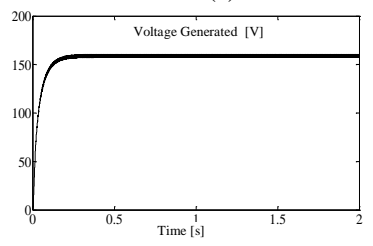

(c)

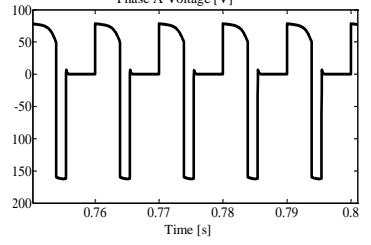

(b)

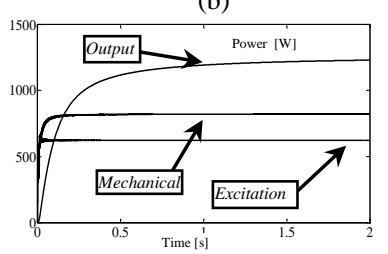

(d)
Figure 4. Current in Phase A (a) - Voltage in Phase A

(b) - Voltage Generated (c) - Power (d)

It can be seen in Figures 3(c) and 4(c) that the generated voltage grows exponentially in the transient at the beginning of the simulation due to the capacitor loading which is used in filtering the voltage produced by the generator, featuring a pulsed characteristic due to phase switching. It can be noticed that with the excitation voltage growth the generated voltage increases significantly. The same behavior appears in the phase current curves of the machine shown in Figures 3(a) and 4(a).

The peak value of current with increased excitation voltage is substantially higher. Regarding the generated power shown in Figures 3(d) and 4(d) it is noticed that it can be raised by increasing the excitation power. Thus it can be verified that using the excitation voltage as a strategy to control the generated voltage has the disadvantage of requiring larger and more robust excitation voltage sources, increasing the volume, value and weight of the system. 
Another simulation test was performed to verify the SRG behavior with respect to the variation of the magnetization angles $\theta_{\text {on }}$ and $\theta_{\text {off }}$ of the phases.

Then a set of combinations of $\theta_{o n}$ and $\theta_{\text {off }}$ angles are implemented and the corresponding value of the generated power is obtained. In this test, the speed was maintained constant at $1500 \mathrm{rpm}$ and the DC bus voltage set at $80 \mathrm{~V}$. Figure 5 shows the graph of the generated power as a function of the opening and closing angles of the switches.

It is observed in this figure that the turn-on angle of the switches $\left(\theta_{o n}\right)$ has little influence on the generated power, which decreases slightly when this parameter has small values. On the other hand, the turn-off angle $\left(\theta_{\text {off }}\right)$ has a great influence on the power generated power.

Due to the low influence of the turn on angle $\left(\theta_{\text {on }}\right)$ on the generated power, in the simulation tests presented in the sequence, its value was kept fixed at -3 degrees in relation to the alignment position. Meanwhile, the $\theta_{\text {off }}$ angle was gradually incremented in 3-degrees steps in the range between 15 and 30 degrees.

Figure 6 shows the current curves in phase A of the SRG due to $\theta_{\text {off }}$ variation. It is noticed that the greater the turn off angle, the greater the phase current. For a 30degree turn-off angle the current in the windings reaches peak values near 40A, whereas when that angle is 15 degrees the current is approximately $5 \mathrm{~A}$.

The generated voltage shown in figure 7 also acknowledges that the turn-off angle exerts a strong influence on its amplitude. For 30-degree values the generated voltage reaches approximately $174 \mathrm{~V}$, while for 15 degrees the value of the generated voltage is close to $61 \mathrm{~V}$.

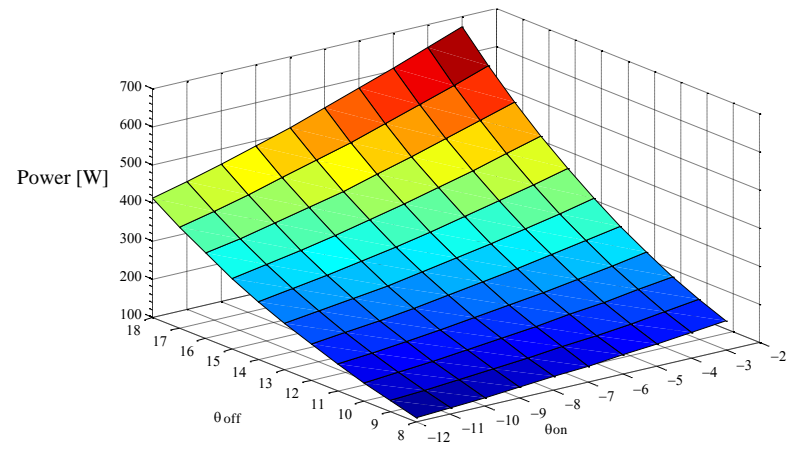

Fig. 5. 3D graph for power generated as a function of the angles $\theta_{o n}$ and $\theta_{\text {off }}$.

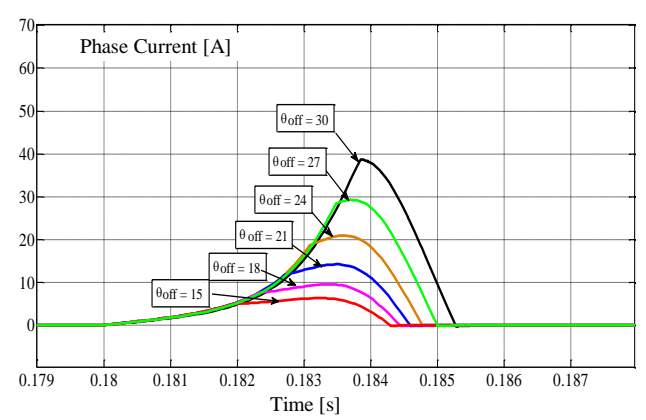

Fig. 6. SRG Phase Current

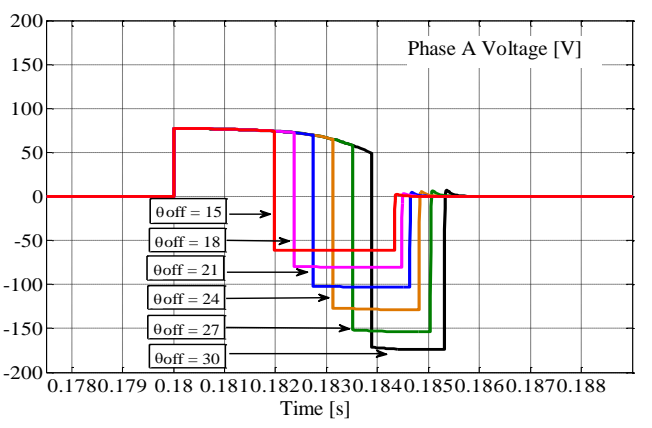

Fig. 7. SRG Phase A Voltage

Table 1 shows the values of $\theta_{\text {off }}$ angle, the current in a phase and the generated voltage at the load.

Table 1 . Phase current and voltage generated by varying $\theta_{\text {off. }}$

\begin{tabular}{|c|c|c|}
\hline$\theta_{\text {off }}($ Degree $)$ & Phase Current (A) & $\begin{array}{c}\text { Voltage Generated } \\
(\mathrm{V})\end{array}$ \\
\hline 15 & 6,5 & 61,5 \\
\hline 18 & 9,8 & 80 \\
\hline 21 & 14,3 & 102 \\
\hline 24 & 21 & 127,8 \\
\hline 27 & 29,2 & 154,2 \\
\hline 30 & 38,7 & 174 \\
\hline
\end{tabular}

Figure 8 shows the curves for the magnetization flux in phase A of the SRG with the increase of the excitation voltage. It is perceived that the higher the amplitude of the excitation voltage the greater the area of the magnetization loop and consequently the higher the voltage generated.

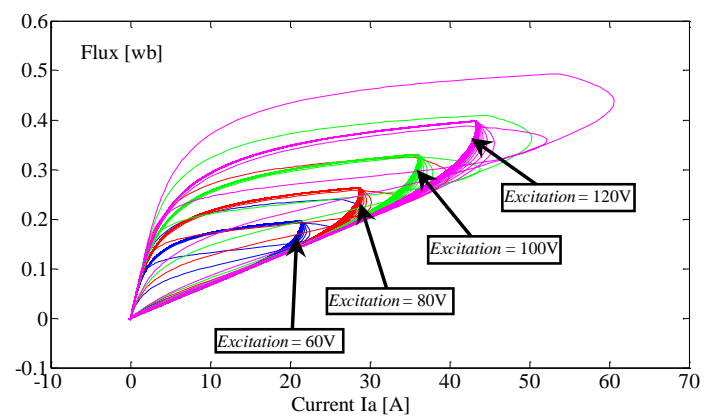

Fig. 8. Magnetizing flux for phase A considering several values for the excitation voltage.

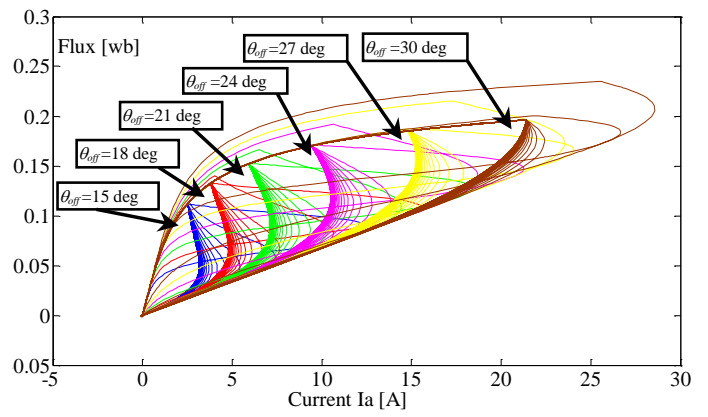

Fig.9. Magnetizing flux for phase A considering several values for angle $\theta_{o f f}$ of the switches. 
However as predicted this practice requires excitation power supplies with higher power raising the implementation costs. Thus the increase in generated voltage from the variation of the switches turn-off angle is a viable alternative. Figure 9 shows the magnetization curves in phase A of the SRG according with $\theta_{\text {off }}$ variation.

This test was accomplished by setting the excitation voltage value to $60 \mathrm{~V}$ and the mechanical shaft speed at $1500 \mathrm{rpm}$. It is noticed that the greater the turn off angle the greater the magnetization loop and consequently the greater the generated voltage.

It can be perceived that keeping fixed the excitation and varying the magnetization angles may be an advantageous technique under the aspect that it does not require additional high power sources to excite the generator.

Another advantage is that it can easily be manufactured using analog or digital electronics, being quite attractive from the point of view of low production cost.

\section{Experimental Results}

To validate the simulation results an experimental platform was developed. The platform consists of a 6HP, 6/4 SRG coupled to a three-phase induction motor driven by a frequency inverter.

An uncontrolled rectifier bridge with a $20 \mathrm{mF}$ capacitor was used to provide the input power of the AC network. The variable voltage source was used to adjust the excitation voltage. The HB converter was assembled with the 1200 V/50A IGBT modules.

A circuit board for $\theta_{\text {off }}$ angle adjustment was designed with analog circuits. The experimental platform picture is shown in Figure 10.

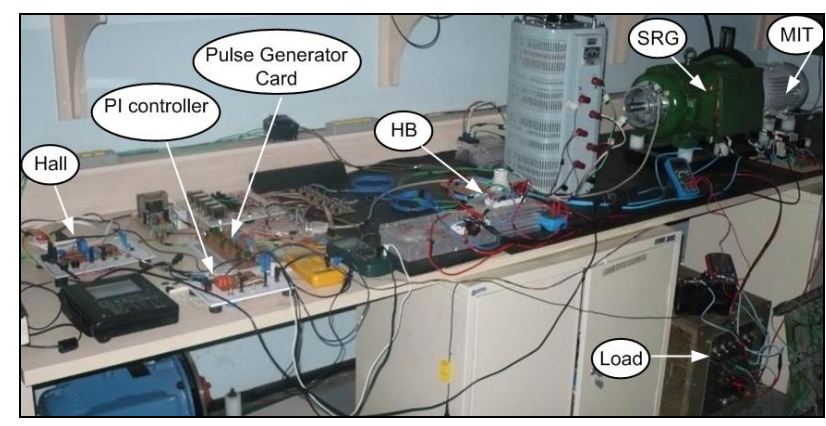

Fig. 10. Experimental Platform

In the results shown in Figures 11(a) and 11(b) the machine speed is kept fixed at 1500rpm, the load resistance is $45 \Omega$ and the excitation voltage is $50 \mathrm{~V}$.

The current in the machine windings reaches the peak of 24A. As shown in Figure 11(b) the value of the generated voltage is approximately $150 \mathrm{~V}$. The current flowing through the load in this experiment is approximately $3.4 \mathrm{~A}$.

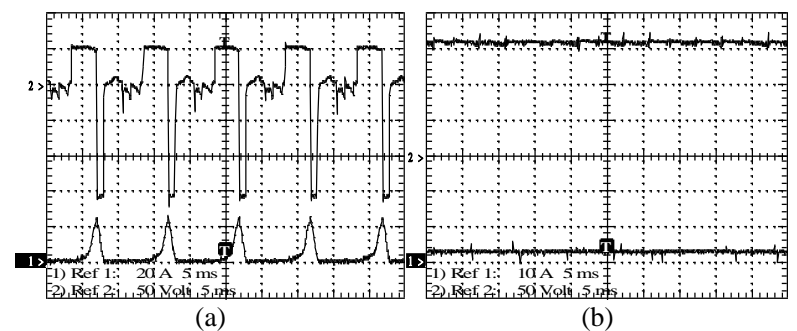

Fig. 11. (a) - Phase A Voltage (upper) and Phase A Current (lower); (b) - Voltage Generated (upper) and Load Current (lower)

In the results shown in Figure 12(a), the excitation voltage has the value of $100 \mathrm{~V}$. This increase in excitation directly influences the current value in the generator phase and considerably increases the values of the generated voltage and current in the load.

As shown in Figure 12 (a) the current circulating in phase $\mathrm{A}$ of the generator reaches about 48A. According to Figure 12(b) the generated voltage reaches $360 \mathrm{~V}$ and the current through the load resistance reaches approximately $6 \mathrm{~A}$.

Figures 13 and 14 show the results for a fixed value of the excitation voltage but varying the rotational speed of the machine. The purpose of this test is to verify the dependence of the generated voltage with respect to the mechanical speed of the shaft.

The excitation voltage is constant at a value of $50 \mathrm{~V}$. The shaft rotation speed is adjusted at 1800rpm in Figure 13 and 2200rpm in Figure 14.

According to Figures 13 (a) and 14 (a) (lower trace), the current in the machine windings presents very close values. Another conclusion is that the generated voltage and the current flowing through the load, illustrated in Figures 13(b) and 14(b) have similar amplitudes. Thereby it can be asserted that in this operating range the effect of the rotation speed on the value of the generated voltage is very low.

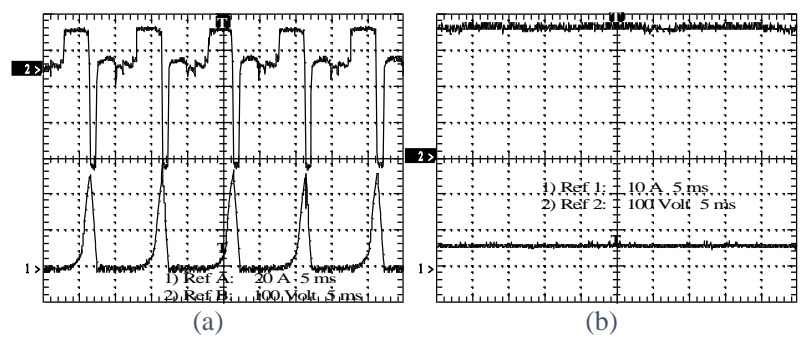

Fig. 12. (a) - Phase A Voltage (upper) and Phase A Current (lower); (b) - Voltage Generated (upper) and Load Current (lower)

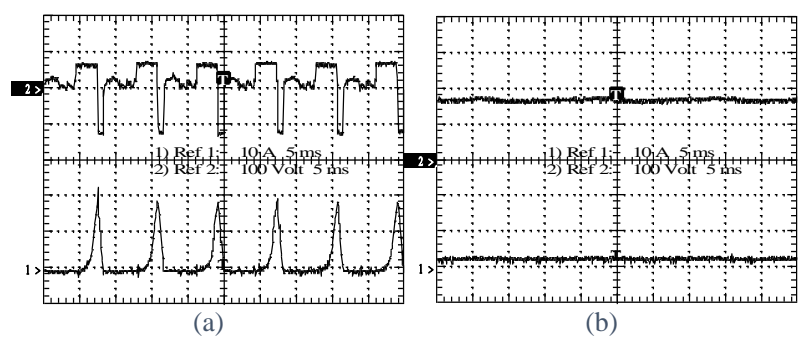

Fig. 13. (a) - Phase A Voltage (upper) and Phase A Current (lower); (b) - Voltage Generated (upper) and Load Current (lower) 


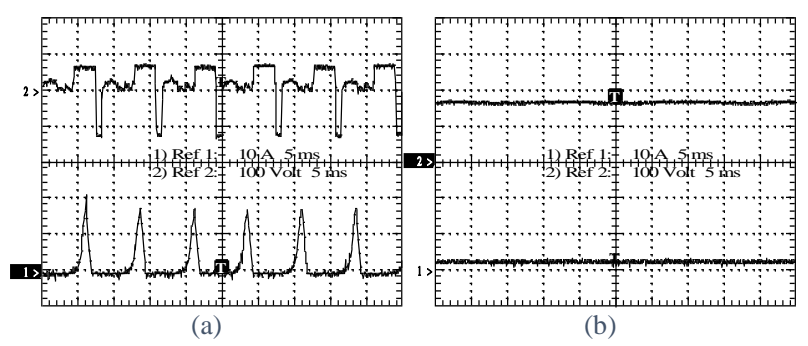

Fig. 14. (a) - Phase A Voltage (upper) and Phase A Current (lower); (b) - Voltage Generated (upper) and Load Current (lower)

Figure 15 shows the results obtained by setting the excitation voltage at $80 \mathrm{~V}$ and the shaft speed at $1500 \mathrm{rpm}$ and gradually changing the $\theta_{\text {off }}$ angle of the switches. In both the voltage and current graphs the initial turn-off angle is 15 degrees, varying gradually by 3 degrees up to 30 degrees.

It is observed in Figure 15(b) that for $\theta_{\text {off }}=30^{\circ}$ the current in the windings reaches approximately 40A, whereas when that angle is $15^{0}$ the current drops dramatically to $5 \mathrm{~A}$. With this, it is observed that the higher the turn off angle, the higher the current at the generator phases and hence the higher the generated voltage.

Figure 15(a) shows the phase voltage. For an angle $\theta_{\text {off }}$ $=30^{\circ}$ the generated voltage is about $170 \mathrm{~V}$. When the turn off angle $\theta_{\text {off }}=15^{\circ}$ the generated voltage reaches $60 \mathrm{~V}$.

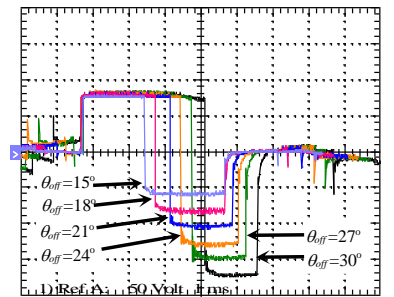

(a)

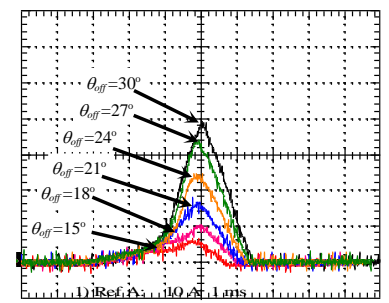

(b)

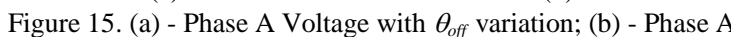
Current with with $\theta_{\text {off }}$ variation.

\section{Acknowledgement}

The authors would like to thank IFG for the financial support.

\section{References}

[1] Powell, D.J.; Jewell, G.W.; Calverley "Iron loss in a modular rotor switched reluctance machine for the "MoreElectric" aero-engine" Magnetics, IEEE Transactions on Oct. 2005, vol. 41.

[2] Cardenas,R. at. al. "Control of a switched Reluctance Generator for Variable-Speed Wind Energy Applications" Trans. on Energy Convertion, Vol. 20, 2005.

[3] Bao, Y.J.; Cheng, K.W.E. "Research on a novel switched reluctance generator for wind power generation" IEEE PESA, 2011.

[4] Sozer ,Y. and Torrey, D. A. "Closed Loop Control of Excitation Parameters for High Speed Switched-Reluctance Generators", IEEE Transactions On Power Electronics, Vol. 19, No. 2, March 2004.

[5] Viajante, G.P.et all, "Estratégia para Melhoria da Eficiência da Conversão Eletromecânica de Energia do GRV" Eletrônica de Potência, v. 16, p. 367-375, Novembro 2011.

[6] Silveira, A. W. F. V., Andrade, D. A., Fleury, A., Gomes, L. C. Bissochi C. A., de Paula, H. "Generated Voltage Control In A Switched Reluctance Motor/Generator" Eletrônica de Potência, v. 15, n. 3, p. 203-212, jun./ago. 2010.

[7] Chen, H., Jason J. G, "Implementation of the Three-Phase Switched Reluctance Machine System for Motors and Generators" IEEE Transactions On Mechatronics, Vol. 15, No. 3, June 2010.

[8] Fleury, A. V. S., Silva F. S., Araújo, W. R. H., Andrade ,D.A., Silveira ,A. W. F. V. "Reduced switch count converter for switched reluctance generators" Eletrônica de Potência, Vol. 13, no. 3, Agosto 2008.

\section{Conclusion}

The SRG dynamic performance analysis was presented. The mathematical model of the machine was developed and the magnetic circuit saturation was included from the phase inductance representation by Fourier series expansion. The experimental and simulation results were evaluated considering the SRG driving in three different ways: variable excitation voltage and fixed mechanical shaft velocity, fixed excitation voltage and variable rotor speed, and finally varying the turn-off angle $\left(\theta_{\text {off }}\right)$. From the obtained results, it has been verified that increasing the machine excitation voltage it is possible to raise the output power delivered to the load. However, depending on the size of the generator used this technique becomes impractical given the growth in implementation costs with the need for high power sources. Thus, the strategy of varying the turn-off angle of the switches has proven to be a feasible alternative. 